\title{
Advancements in the HELIAS 5-B breeding blanket structural analysis
}

\section{G. Bongioví ${ }^{1}$, A. Häußler ${ }^{1}$ and the W7-X team}

${ }^{1}$ Karlsruhe Institute of Technology, INR, Germany

Within the framework of EUROfusion consortium, the work package S2 aims at developing the HELIcal-axis Advanced Stellarator (HELIAS) as a possible long-term alternative to a tokamak DEMO. Researches are currently ongoing at KIT in order to attain a preliminary design of the HELIAS 5-B breeding blanket (BB), taking into account as initial input the design experience acquired in the pre-conceptual design phase of the tokamak DEMO BB. To this end, the Helium-Cooled Pebble Bed (HCPB) and the Water Cooled Lithium Lead (WCLL) BB concepts have been considered, focusing on the investigation of the suitability of their main structural features to the stellarator geometry. In this regard, possible design constraints coming from the Remote Maintenance have to be fulfilled in order to better orient the blanket segmentation. In the present work, a more sophisticated assessment of the BB modules structural behavior has been performed. Attention has been also paid to the refinement of the numerical models so far adopted, investigating the impact of the major assumptions, such as Vacuum Vessel temperature and equivalent Young's Modulus, on the obtained results. To this purpose, dedicated parametric assessment has been carried out and discussed in this work.
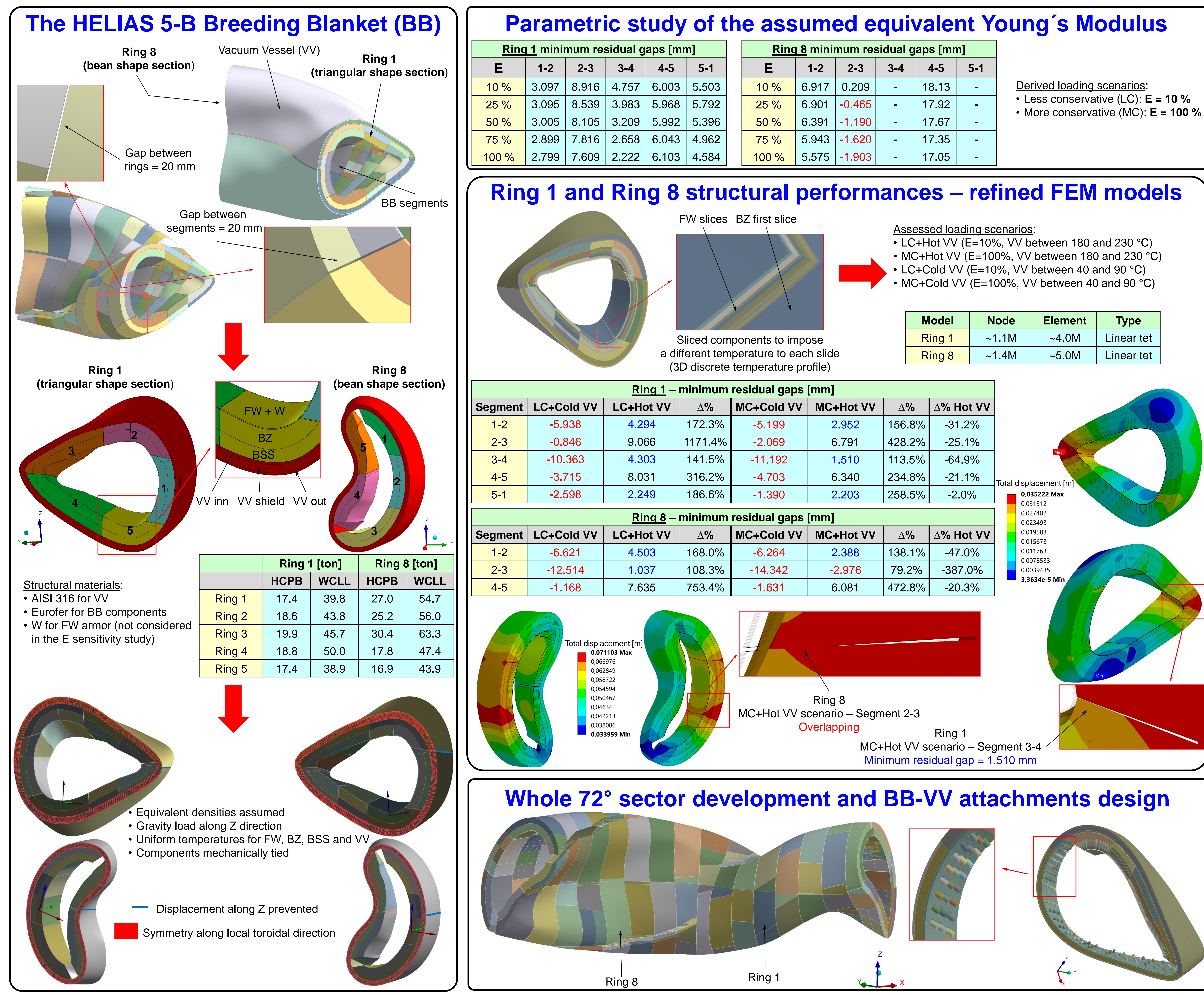

Ring 1 and Ring 8 structural performances - refined FEM models
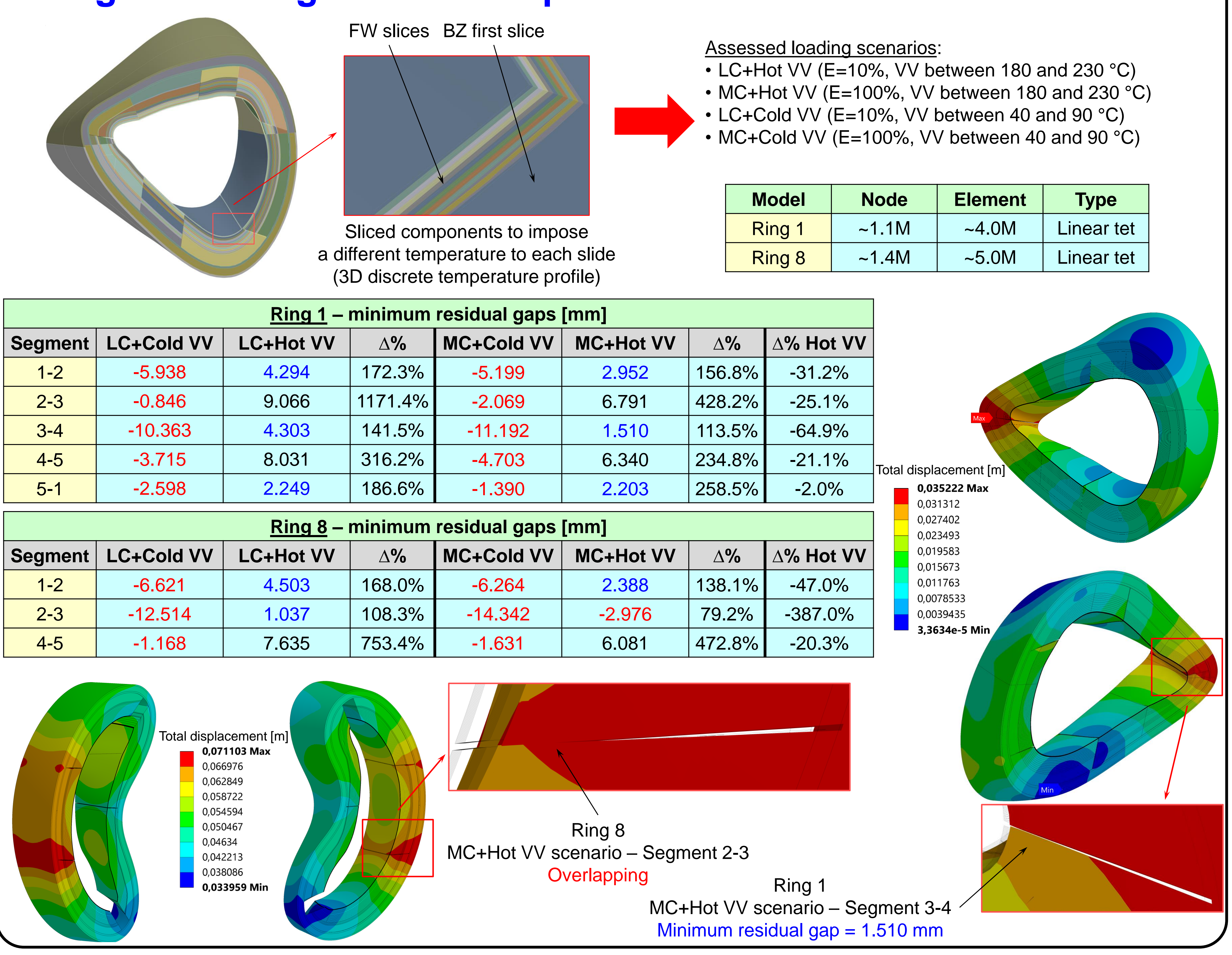

Whole $72^{\circ}$ sector development and BB-VV attachments design
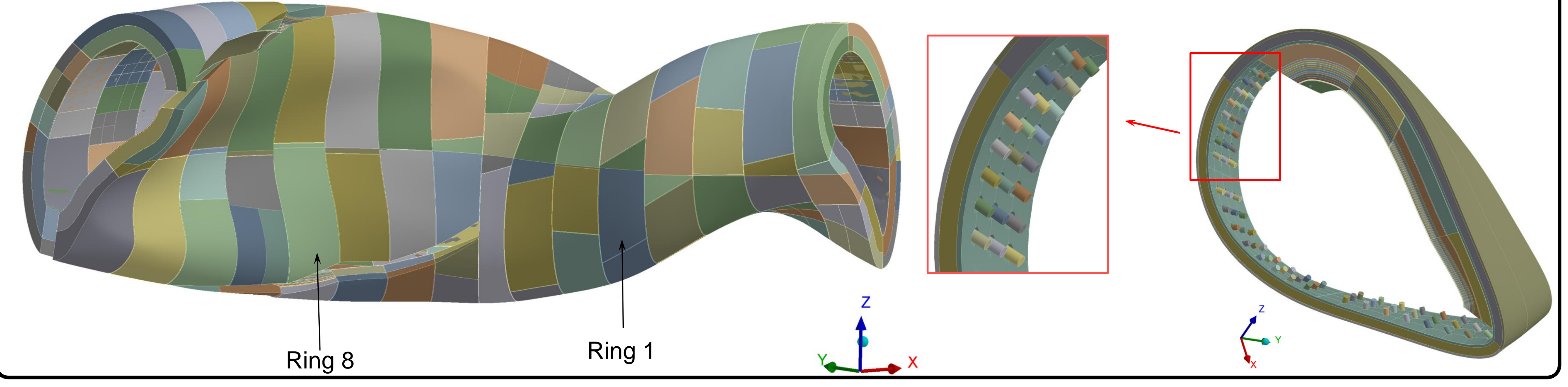

\section{Conclusion}

Structural calculations of the most representative BB rings have been performed adopting detailed homogenized FEM models. The quite promising outcomes encourage the follow-up of the activity aimed at assessing a whole BB sector, which extends for $72{ }^{\circ}$ toroidally. Moreover, since a strong influence of the VV temperature on the BB structural performances has been found out, the development of an attachment system devoted to connect BB and VV is crucial, together with the design of the BB segments internal components, to more realistically assess the HELIAS 5-B BB thermomechanical behavior. Lastly, the RM-BB interface should be defined.

\section{EUROfusion}

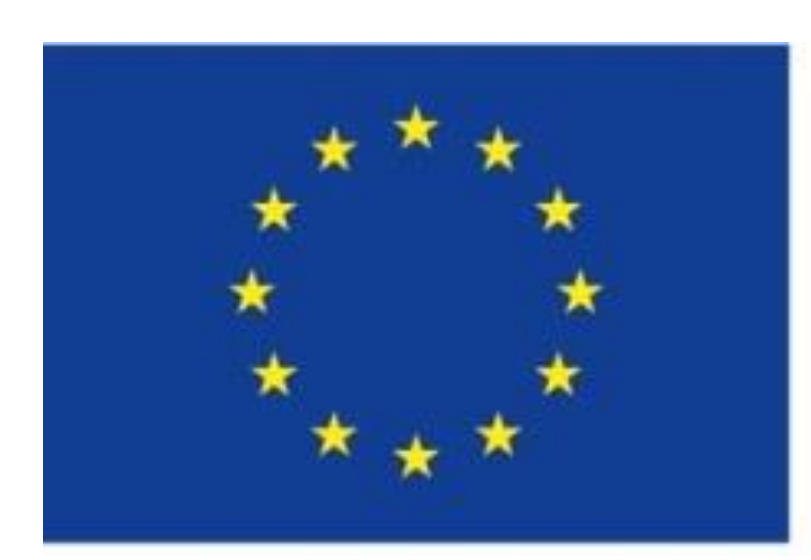

This work has been carried out within the framework of the EUROfusion Consortium and has received funding from the Euratom research and training programme 2014-2018 and 2019-2020 under grant agreement No 633053. The views and opinions expressed herein do not necessarily reflect those of the European Commission. 